\title{
Superior Thermoelectric Performance in PbTe-PbS Pseudo-binary: Extremely Low Thermal Conductivity and Modulated Carrier
}

\section{Concentration}

Di Wu, ${ }^{1,5, a}$ Xiao Tong, ${ }^{1, a}$ Li-Dong Zhao, ${ }^{2}$ Wei Li, ${ }^{1,5}$ Lijun Wu, ${ }^{3}$ Qing Tan, ${ }^{4}$ Yanling Pei, ${ }^{2}$ Li Huang, ${ }^{1,5}$ Jing-Feng $\mathrm{Li}^{4}{ }^{4}$ Yimei Zhu, ${ }^{3}$ Jiaqing $\mathrm{He}^{1,5^{*}}$

${ }^{1}$ Department of Physics, South University of Science and Technology of China, Shenzhen 518055, China

${ }^{2}$ School of Materials Science and Technology and Engineering, Beihang University, Beijing 100191, China

${ }^{3}$ Condensed Matter Physics and Materials Science Department, Brookhaven National Laboratory, Upton 11973, USA

${ }^{4}$ Key Laboratory of New Ceramics and Fine Processing, School of Materials Science and Eng ineering, Tsinghua University, Beijing, 100084, China

${ }^{5}$ Shenzhen Key Laboratory of Thermoelectric Materials, Shenzhen, 518055, China

${ }^{\mathrm{a}}$ These authors contributed equally to this work

* To whom correspondence should be addressed. Email: hejq@sustc.edu.cn

Lead chalcogenides have exhibited their irreplaceable role as thermoelectric materials at the medium temperature range, owing to highly degenerate electronic bands and intrinsically low thermal conductivities. PbTe-PbS pseudo-binary has been paid extensive attentions due to the even lower thermal conductivity which originates largely from the coexistence of both alloying and phase-separated precipitations. To investigate the competition between alloying and phase separation and its pronounced effect on the thermoelectric performance in $\mathrm{PbTe}-\mathrm{PbS}$, we systematically studied Spark Plasma Sintered (SPSed) 3 at\% Na- doped $(\mathrm{PbTe})_{1-x}(\mathrm{PbS})_{x}$ samples with $x=10 \%, 15 \%, 20 \%, 25 \%, 30 \%$ and $35 \%$ by means of transmission electron microscopy (TEM) 
observations and theoretical calculations. Corresponding to the lowest lattice thermal conductivity as a result of the balance between point defect- and precipitates- scattering, the highest figure of merit $Z T \sim 2.3$ was obtained at $923 \mathrm{~K}$ when PbS phase fraction $x$ is at $20 \%$. The consistently lower lattice thermal conductivities in SPSed samples compared with corresponding ingots, resulting from the powdering and follow-up consolidation processes, also contribute to the observed superior ZT. Notably, the onset of carrier concentration modulation $\sim 600 \mathrm{~K}$ due to excessive Na's diffusion and re-dissolution leads to the observed saturations of electrical transport properties, which is believed equally crucial to the outstanding thermoelectric performance of SPSed PbTe-PbS samples.

\section{Introduction}

Thermoelectric materials can directly harvest electricity from waste heat and realize small-scale refrigerating, thus have drawn growing research interests for decades. ${ }^{1}$ The performance of a specific thermoelectric material is evaluated by a dimensionless figure of merit $Z T=S^{2} \sigma \mathrm{T} / \kappa$, where $\mathrm{S}$ is the Seebeck coefficient, $\sigma$ is the electrical conductivity, $\mathrm{T}$ is the absolute temperature and $\kappa$ is the thermal conductivity. To achieve a decent thermoelectric performance, a thermoelectric material shall own proper electronic structures to assure a high power factor $P F=\mathrm{S}^{2} \sigma$ and simultaneously a low thermal conductivity $\kappa$. The family of lead chalcogenides $\mathrm{PbQ}$ ( $\mathrm{Q}=\mathrm{Te}$, Se or $\mathrm{S}$ ) are such ideal thermoelectric materials. The high band degeneracies ${ }^{2,3}$ assure high effective masses favorable for high Seebeck coefficients while the narrow band gaps ${ }^{2,4}$ indicate decent electrical conductivities can be achieved via doping; meanwhile, the intrinsic lattice thermal conductivities of PbQ are astonishingly low considering their simple crystal structures 
(space group $F m \overline{3} m$ ), thanks to the high anharmonicity of the bonds and recently identified soft behaviors of the lattice ${ }^{5,6}$. The power factors of lead chalcogenides can be further enhanced by band structure engineering via introducing resonant states, i.e., Tl doped $\mathrm{PbTe}^{7,8}$, or tuning band degeneracy through alloying, i.e., PbSe alloyed $\mathrm{PbTe}^{9}, \mathrm{MgTe}$ alloyed $\mathrm{PbTe}^{10}$, PbSe-PbS alloys ${ }^{11}$, and $\mathrm{MnTe}$ alloyed $\mathrm{PbTe}^{12}$. The lattice thermal conductivity can be reduced by various nanostructuring techniques, e.g., in-situ nano-phase precipitations ${ }^{10,13-15}$ and ex-situ powdering processes $^{16-18}$ (nano-inclusions or grain refining). Meanwhile, the pseudo-binary systems

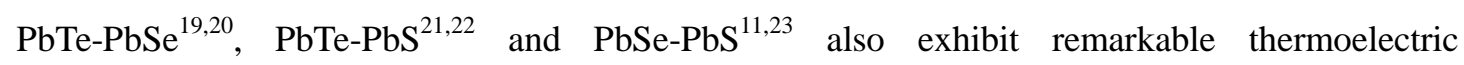
performances. PbTe-PbSe and PbSe-PbS are both believed to be complete solid solution, and their low thermal conductivities results from the alloy scattering due to Te/Se and Se/S point defects, although recent transmission electron microscopy studies disclosed that nano-precipitates did exist in PbSe-PbS alloys ${ }^{23}$. In contrast, PbTe-PbS system has a verified immiscible gap between PbTe and $\mathrm{PbS}$ phases $^{24}$, and thus exhibits phase separation either in the form of nucleation and grain growth processes or spinodal decompositions ${ }^{25,26}$, or an intermediate case ${ }^{27}$, depending on the relative phase fraction. The competition between alloying and phase separation between PbTe and $\mathrm{PbS}$ leads to extremely complex microstructures of $\mathrm{PbTe}-\mathrm{PbS}$ system, which results in the very low lattice thermal conductivities in this binary as compared with each of pristine $\mathrm{PbTe}$ or $\mathrm{PbS}$.

To investigate the microstructure evolvement as $\mathrm{PbS}$ fraction and optimize the thermoelectric performance, we conducted a systematical study on 3 at\% Na- doped $(\mathrm{PbTe})_{1-x}(\mathrm{PbS})_{x}$ SPSed samples ( $x=10 \%, 15 \%, 20 \%, 25 \%, 30 \%$ and $35 \%)$, and obtained consistently lower lattice thermal conductivity values than as well as comparative power factors with those of corresponding ingots $^{21}$. Since the high-energy powdering procedures and follow-up consolidation processes tend 
to introduce extra point defects and interfacial potential between grains, the obtained results reflect a trade-off between reduced lattice thermal conductivity and reduced carrier mobility. We found the lowest thermal conductivity belongs to a nominal PbS phase fraction of $20 \%$, which could be attributed to the least complete phase separation ${ }^{27}$ and the most effective precipitates scattering as discussed in our later calculations. The phase separations between PbTe and PbS not only evolve according to their relative phase fractions, but are also largely affected by the heat treatment history ${ }^{27,28}$; therefore, we have performed strictly the same heat treatment procedures for all the samples. What is more, the diffusion and re-dissolution of excessive Na into PbTe-PbS matrix grains over $\sim 600 \mathrm{~K}$ lead to the increment of hole concentration, and are suggested to be responsible for the observed saturations of Seebeck coefficients and electrical conductivities. This phenomenon of carrier concentration modulation with temperature is believed crucial to maintain the superior thermoelectric performance at high temperatures in the Na- doped PbTe-PbS system.

\section{Experimental results and discussions}

Thermoelectric properties of 3 at\% Na- doped $(\mathrm{PbTe})_{1-x}(\mathrm{PbS})_{x}$ series are plotted as shown in Figure 1. The electrical conductivities $\sigma$ of all the SPSed samples decrease as elevating temperature from $300 \mathrm{~K}$ to $600 \mathrm{~K}$, and then show the sign of saturations once temperature goes above 600 K, Figure 1(a). The Seebeck coefficients S, Figure 1(b), increase almost linearly from $\sim 60-65 \mu \mathrm{V} \mathrm{K}^{-1}$ at $300 \mathrm{~K}$ to $\sim 260-280 \mu \mathrm{V} \mathrm{K}^{-1}$ at $600 \mathrm{~K}$, where they start to decrease abruptly in a mild manner. The saturation behaviors of both electrical conductivity and Seebeck coefficient above $600 \mathrm{~K}$ in these SPSed samples are dissimilar to the normally reported Na- doped $\mathrm{PbTe}^{21,29}$, $\mathrm{PbS}^{30,31}, \mathrm{PbTe}-\mathrm{PbSe}^{20}$ or K- doped $\mathrm{PbTe}-\mathrm{PbS}^{22}$, whose $\sigma$ and $\mathrm{S}$ all showed smooth and gradual 
variations as temperature. The abrupt saturation is suggested to result from the diffusion and re-dissolution of Na which is confined at grain/phase boundaries at low temperature into the grain matrix at high temperature, as will be discussed more carefully in a later section. The $\sigma$ values of $(\mathrm{PbTe})_{1-x}(\mathrm{PbS})_{x}$ SPSed samples decrease systematically as the content of PbS $x$ increases from $10 \%$ to $35 \%$, while corresponding S values vary little. Except for the sample with $x=35 \%$, all $(\mathrm{PbTe})_{1-x}(\mathrm{PbS})_{x}$ samples exhibit very similar power factors $(P F)$, especially at high temperatures, Figure 1(c). The peak values of $P F s$ are located at $623 \mathrm{~K}$ and vary from $\sim 23.7$ to $26.5 \mu \mathrm{W} \mathrm{cm}{ }^{-1} \mathrm{~K}^{-2}$, which are comparable to the highest reported values ${ }^{9,14,21}$ in pristine PbTe systems. The total thermal conductivities of different compositions are surprisingly close to each other, all showing a decreasing trend before $700 \mathrm{~K}$ where the upturning starts, Figure 1(d). Each lattice thermal conductivity is obtained by subtracting the electrical contribution from total thermal conductivity, following the Wiedemann-Franz Law as $\kappa_{\text {latt }}=\kappa-\kappa_{\text {ele }}$, where $\kappa_{\text {ele }}=L \sigma T$ in which Lorenz number $L$ can be calculated from reduced Fermi energy $\varepsilon_{\mathrm{F}}$ derived by experimental Seebeck values as described elsewhere ${ }^{32,33}$. It is seen in Figure 1(e) that the lattice thermal conductivity is gradually suppressed with the increase of PbS content till $x=20 \%$, whereafter it starts to increase. It will be seen later that the observed lattice thermal conductivities for different PbS phase fraction $x$ are closed related with their individual detailed microstructures. The lattice thermal conductivity values range from 1.1 to $1.5 \mathrm{~W} \mathrm{~m}^{-1} \mathrm{~K}^{-1}$ at room temperature (303 $\mathrm{K}$ ), and decrease to as low as $\sim 0.38-0.45 \mathrm{~W} \mathrm{~m}^{-1} \mathrm{~K}^{-1}$ at $923 \mathrm{~K}$. The high temperature values of lattice thermal conductivity values are so low that they even approaches the "glass limit" $\sim 0.36 \mathrm{~W} \mathrm{~m}^{-1} \mathrm{~K}^{-1}$ for bulk PbTe system as calculated by Cahill et al. ${ }^{34}$ The high power factors over a broad temperature range (from $450 \mathrm{~K}$ to $923 \mathrm{~K})$ together with the extremely low thermal conductivities result in a high $Z T(>1.5)$ plateau in 
all samples from $600 \mathrm{~K}$ to $923 \mathrm{~K}$ (Figure 1(f)), thus indicating outstanding theoretical thermoelectric efficiencies ${ }^{22}$ that are highly potential for commercial applications. Notably, a maximal $Z T$ of $\sim 2.3$, one of the highest values ${ }^{14,22,35,36}$ among bulk thermoelectric materials, was achieved in 3 at $\%$ Na- doped $(\mathrm{PbTe})_{1-x}(\mathrm{PbS})_{x}$ at $923 \mathrm{~K}$ for $x=20 \%$.
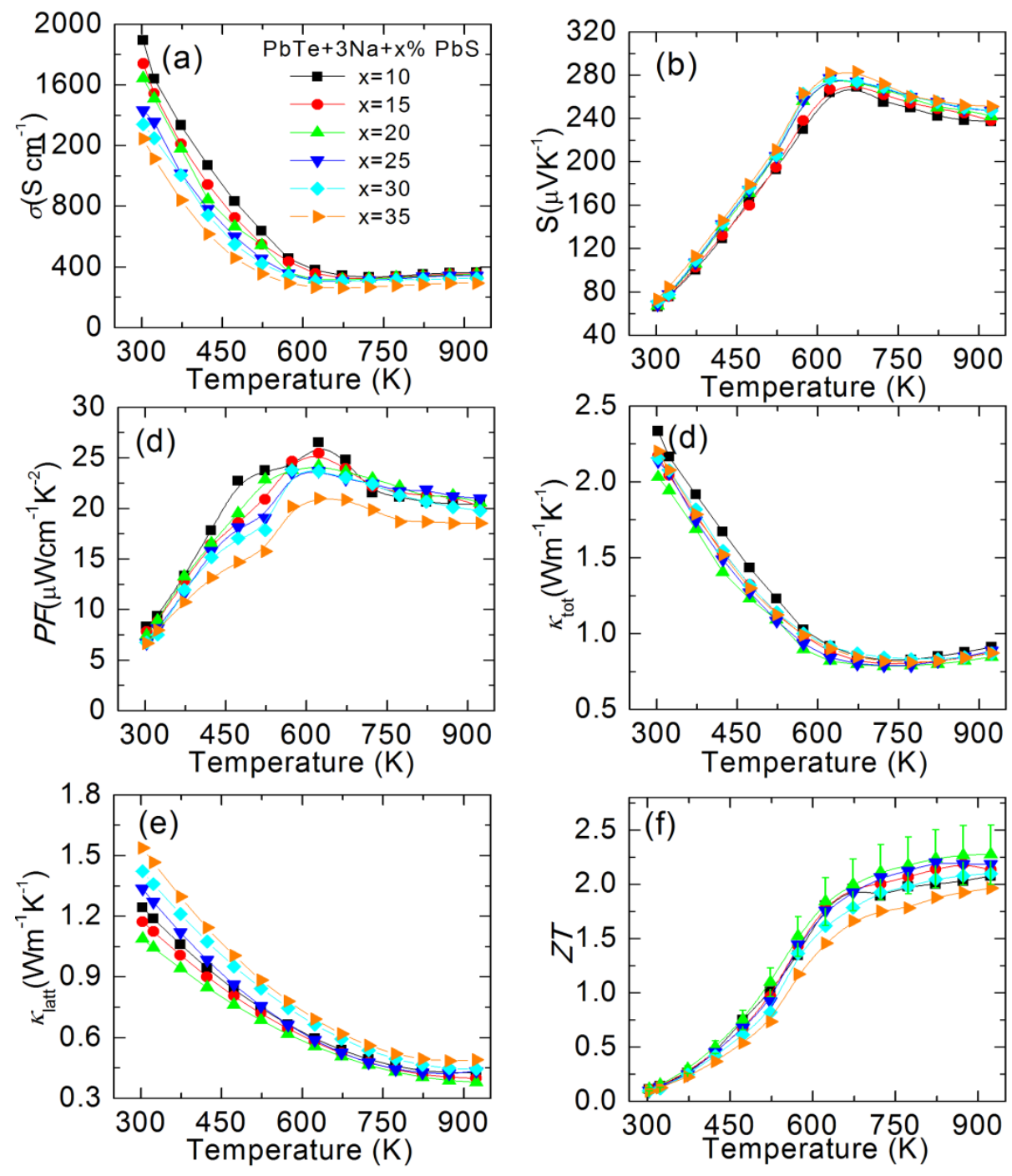

Figure 1 (a) Electrical conductivities, (b) Seebeck coefficients, (c) power factors, (d) total thermal conductivities, (e) lattice thermal conductivities and (f) figure of merit ZTs of 3 at\% Na doped $\mathrm{PbTe}_{1-x} \mathrm{~S}_{x}$ composites with $x=10 \%, 15 \%, 20 \%, 25 \%, 30 \%$ and 35\% respectively. For concision, error bars of $Z T$ was implemented for the $x=20 \%$ sample.

\section{Microstructure analysis}

To explore the underlying physical mechanisms of the observed thermoelectric properties, we 
looked through the microstructural evolution of the 3 at $\%$ Na- doped $\mathrm{PbTe}_{1-x} \mathrm{~S}_{x}(x=10 \%, 20 \%$ and 30\%) specimens with PbS fraction $x$. 10\% PbS sample exhibits high number density nanoscale precipitates with an average size of $\sim 9 \mathrm{~nm}$, as shown in Figure 2(a). The inserted electron diffraction (ED) pattern, covering a region with both precipitates and matrix, reveals a nearly single set of reflections; this indicates the small compositional and weak structural difference between the nanoscale precipitates and the matrix. The statistical volume fraction of the precipitates ( 23\%) is much higher than that of the nominal PbS percentage of $10 \%$, implying that the precipitates are not completely phase-separated $\mathrm{PbS}$ phase. Both evidences suggest the precipitated particles are slightly PbS-richer than the matrix, thus the mass density difference between the precipitations and matrix is considerably smaller than that between pure $\mathrm{PbS}$ and PbTe phase. For this reason, phonon scattering from these precipitates is very weak (shown in a later section) despite of the high number density and small size. Cubical precipitates were widely (118 nm, on average) seen in the 20\% PbS sample, Figure 2(b) and 2(c). The inserted ED pattern was obtained with the aperture including the precipitate and the matrix. The obtained two sets of split reflection ED patterns were indexed to be $\mathrm{PbTe}$ and $\mathrm{PbS}$ separately, implying that the cubical precipitates are the completely phase-separated PbS. Besides, the composition variance between the precipitates and the matrix was also reflected directly by the Z-contrast difference in STEM-HAADF (high-angle annular dark-field) image (Figure 2(c)). Much larger precipitates (ranging from 200 to $800 \mathrm{~nm}$, with an average $\sim 340 \mathrm{~nm}$ ) with irregularly round shape were found in the 30\% PbS sample, Figure 2(d); this, together with the split ED patterns as inserted, provide strong evidence for complete $\mathrm{PbS}$ phase separation. Comparing the relatively large precipitates size with the typical wavelength of phonons which dominate the thermal transport, we don't 
expect an effective reduction of lattice thermal conductivity due to precipitates scattering. We didn't see obvious spinodal decomposition in the $30 \% \mathrm{PbS}$ sample, although this $\mathrm{PbS}$ phase fraction corresponds to the spinodal decomposition region. This phenomenon could be explained by the significantly depressed coherent spinodal line as suggested by Doak et al. ${ }^{37}$, who originally included coherency strains in their density functional calculations and concluded that the depression of spinodal line in temperature is so large that spinodal decomposition can only occur at very low temperatures, where it is probably kinetically unstable. Statistical results, Figure 2(e), reveal that the precipitate size increases while number density decreases with the increase of $S$ fraction. Despite that the smallest size and highest number density of the precipitates were found in $10 \% \mathrm{PbS}$ sample, it is the $20 \% \mathrm{PbS}$ sample that exhibits the lowest lattice thermal conductivity; the detailed relationship between microstructures and lattice thermal conductivities will be discussed in our calculations later. It is also noticed that these microstructural features of SPS samples and the evolution with $\mathrm{PbS}$ content resemble what was reported in corresponding ingots $^{21,26}$.
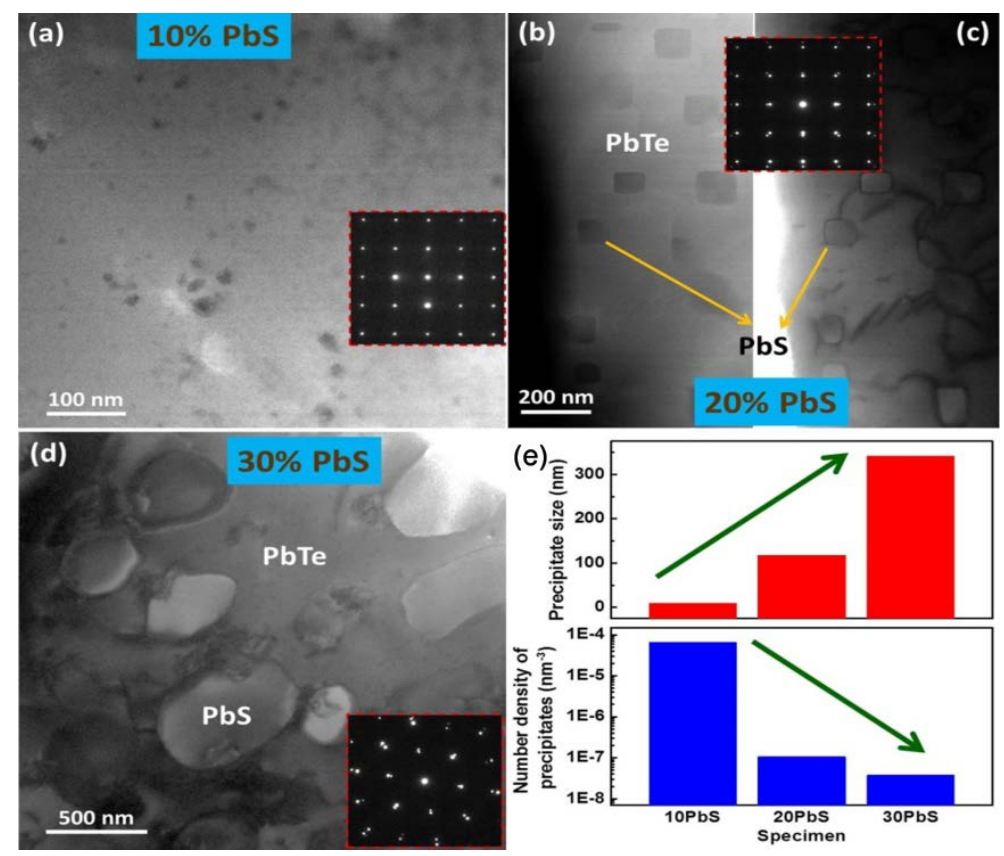

Page 8 / 26 
Figure 2 (a) Low-magnification TEM image of the 10\% $\mathrm{PbS}$ sample reveals nanoscale precipitates with inserted electron diffraction pattern along [100] zone axis; (b) and (c) Low-magnification TEM and STEM-HAADF images of the $20 \% \mathrm{PbS}$ sample exhibit cubical precipitates with inserted electron diffraction pattern along [100] zone axis; (d) Low-magnification TEM image of the $30 \% \mathrm{PbS}$ sample shows large-scale precipitates with inserted electron diffraction pattern along [110] zone axis; (e) the size and number density distribution histograms of precipitates for the $10 \%, 20 \%$, and $30 \%$ PbS samples.

We also performed mediate- or high-resolution STEM-HAADF and STEM-ABF (annular bright-field) techniques on the selected SPS samples ( $x=10 \%, 20 \%$ and $30 \%)$. The interface between the S-rich precipitate (relatively darker in HAADF imaging mode while brighter in ABF imaging mode) and PbTe matrix in the 10\% PbS sample is indistinct, as shown in Figure 3(a) and 3(b), suggesting a weak composition gradient. Such a weak interface and composition contrast can be imagined ineffective to scatter heat-carrying phonons. The cubical precipitates in the $20 \% \mathrm{PbS}$ specimen exhibit regular Moiré fringes in thick areas, which are seen more clearly in ABF image mode, Figure 3(c) and (d). It is well known that Moiré pattern is a kind of diffraction contrast, due to the overlap between two sets of planes (e.g., PbS precipitate and PbTe matrix from top to down) with nearly common periodicity and/or small relative rotation angle. HAADF differentiates from

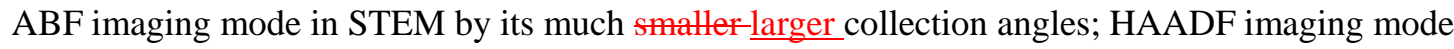
is more sensitive to the composition variation (so called Z-contrast imaging) while ABF exhibit high sensitivity to the diffraction contrast. Clear interfacial contrast and misfit edge dislocations are observed at the straight interfaces around the cubical precipitates, Figure 3(e) and 3(f). As the fraction of $\mathrm{PbS}$ further increases to $30 \%$, much larger $\mathrm{PbS}$ particles then precipitate. It is apparent that the three samples show quite different microstructures, which can be derived from their disparate phase states in the $\mathrm{PbTe}-\mathrm{PbS}$ binary phase diagram: the $10 \% \mathrm{PbS}$ one locates in nucleation and growth region, the $30 \% \mathrm{PbS}$ one lies in spinodal decomposition region, while the 
$20 \% \mathrm{PbS}$ one stays in between. The different microstructures are closely related with transport properties of charge carriers and phonons, which will be carefully treated in our later calculations.

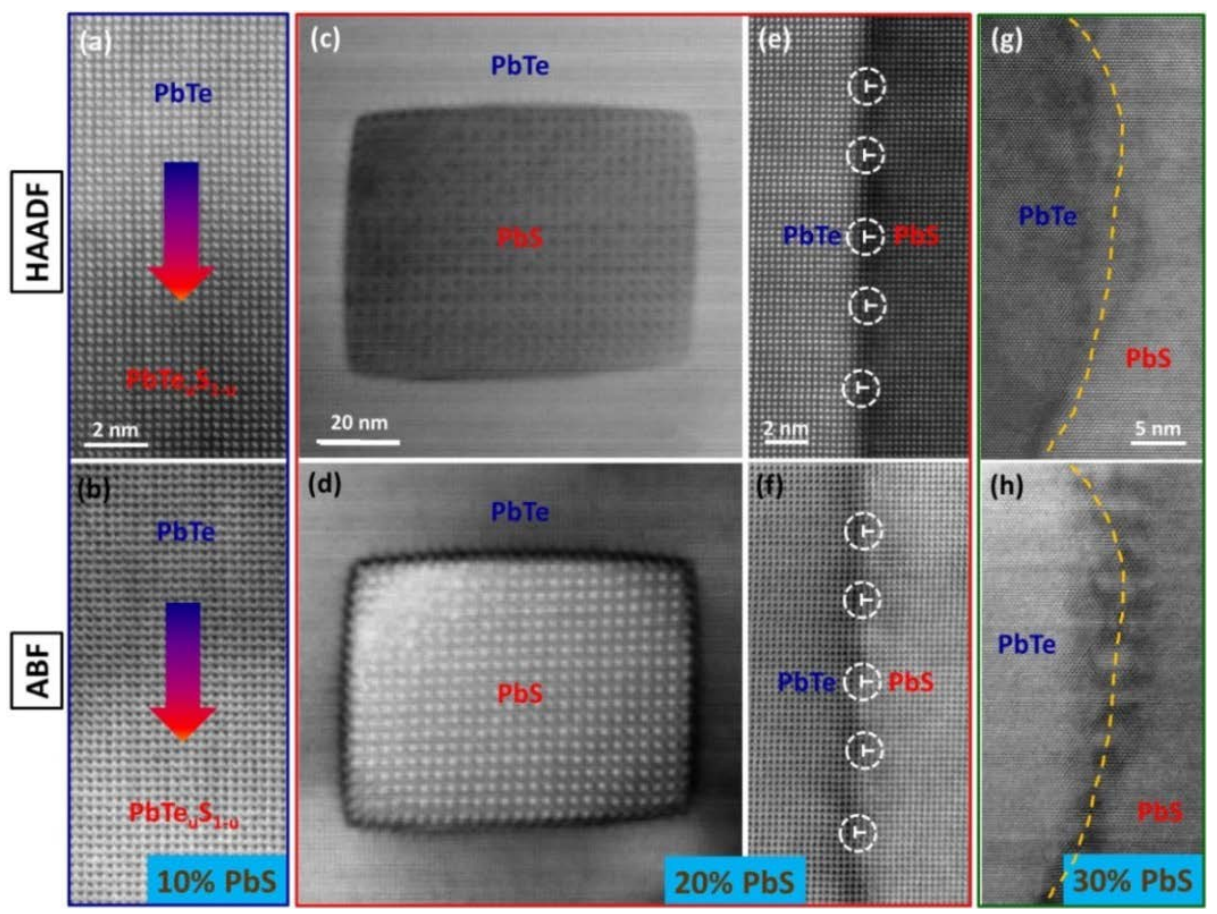

Figure 3 Mediate or high-resolution STEM-HAADF (a, c, e, g) and STEM-ABF (b, d, f, h) images of the 10\% (a, b), 20\% (c-f), and 30\% (g, h) PbS samples: (a) and (b) show the interface between S-rich precipitate and PbTe matrix without clear interface; (c) and (d) shows regular Moiré fringes in PbS cubical precipitate; (e) and (f) reflects the straight interface between the cubical PbS precipitate and the PbTe matrix, with orderly arrayed dislocations; (g) and (h) reveals round interface between the spherical $\mathrm{PbS}$ precipitate and the $\mathrm{PbTe}$ matrix.

\section{Lowest lattice thermal conductivity in $x=20 \%$ SPS sample}

It was seen earlier in Figure 1(e) that the lattice thermal conductivity of 3 at $\%$ Na- doped $(\mathrm{PbTe})_{1-x}(\mathrm{PbS})_{x}$ decreases with $x$ till $20 \%$ and then increases again. To better illustrate this interesting behavior, we re-plot the lattice thermal conductivity vs. $x$ (Figure 4(a)) at $303 \mathrm{~K}, 623$ $\mathrm{K}$ and $923 \mathrm{~K}$, respectively. The nominal composition of $(\mathrm{PbTe})_{0.8}(\mathrm{PbS})_{0.2}(x=0.2)$ exhibits the lowest lattice thermal conductivity consistently at varying temperatures, and the reason is not as 
trivial as it seems from the TEM observations. We ascribe the low lattice thermal conductivity of $(\mathrm{PbTe})_{0.8}(\mathrm{PbS})_{0.2}$ to the relatively higher PbTe-PbS alloying ratio as discussed by Girard et al. ${ }^{21,27}$ together with effective phonon scattering by nanoscale precipitates, and suggest that the incipient nano-precipitates $(\sim 10 \mathrm{~nm})$ in $(\mathrm{PbTe})_{0.9}(\mathrm{PbS})_{0.1}(x=10 \%)$ are relatively weak in scattering phonons despite of their small size.

To elucidate the microstructure-lattice thermal conductivity relation in 3 at $\%$ Na- doped $(\mathrm{PbTe})_{1-x}(\mathrm{PbS})_{x}$ SPS samples, herein we present thermal transport calculations based on revised Callaway model as described elsewhere ${ }^{28}$. As mentioned above, the grain sizes in SPS samples are mesoscale ( $>2 \mu \mathrm{m}$ ), hence the grain boundaries are fairly weak in scattering short and intermediate wavelength phonons which dominate the thermal transport. Moreover, although strains and dislocations might possibly exist at phase/grain boundaries, they are not considered in our calculations since their number densities are not high and hard to be quantified precisely ${ }^{38}$. The contributions from Umklapp process, Normal process, point defects- and precipitates- scattering are included in the calculation; the detailed formula and information can be found in supporting information (SI). In $(\mathrm{PbTe})_{0.9}(\mathrm{PbS})_{0.1}$, the matrix and the nano-precipitates were believed to be sulfur-poor and sulfur-rich respectively; however, as evidenced from TEM observations earlier, the composition difference between precipitations and matrix is quite small. The small composition contrast implies their inefficiency on phonon scattering despite of the precipitates' small size and high number density, as suggested by Eqn. S4-S5 and literatures ${ }^{38,39}$. In the spinodal decomposition region $(x>30 \%)$ of $\mathrm{PbTe}-\mathrm{PbS}$ phase diagram ${ }^{24}$, complete phase separation yields a PbTe-rich $\mathrm{PbTe}_{0.93} \mathrm{~S}_{0.07}$ phase and a PbS-rich $\mathrm{PbS}_{0.98} \mathrm{Te}_{0.02}$ phase. In contrast, $\mathrm{S}$ alloying in $\mathrm{PbTe}$ can be as large as $\sim 0.12$ in the intermediate region ( $x \sim 16 \%$ ) between nucleation-and-growth and 
spinodal decomposition in Na- doped PbTe-PbS composites even after an annealing process, indicating this incompletely phase separated state might be more thermodynamically preferred ${ }^{27,40}$. Our statistics based on TEM observations confirm this argument and show that the volume fraction of PbS-rich precipitates in $(\mathrm{PbTe})_{0.8}(\mathrm{PbS})_{0.2}$ is only $7 \%$ (contrasting to the nominal $~ 20 \%$ $\mathrm{PbS}$ fraction), indicating a high alloying ratio of $\mathrm{PbS}$ as the result of incomplete phase separation. The reliability of our calculations is justified by comparing the calculated results directly with experiments, as shown in Figure 4(b); note that the lattice thermal conductivity were calculated based on the volume fraction of PbTe-rich and PbS-rich phases for $(\mathrm{PbTe})_{0.8}(\mathrm{PbS})_{0.2}$ and $(\mathrm{PbTe})_{0.7}(\mathrm{PbS})_{0.3}$. Under the margin of error, our calculated and experimental data reinforced the validity of our previous argument that $(\mathrm{PbTe})_{0.8}(\mathrm{PbS})_{0.2}$ corresponds to an incomplete phase separation with larger S alloying, and that the small nano-precipitates widely found in $(\mathrm{PbTe})_{0.9}(\mathrm{PbS})_{0.1}$ come from incipient phase separation and cannot effectively scatter heat-carrying phonons.

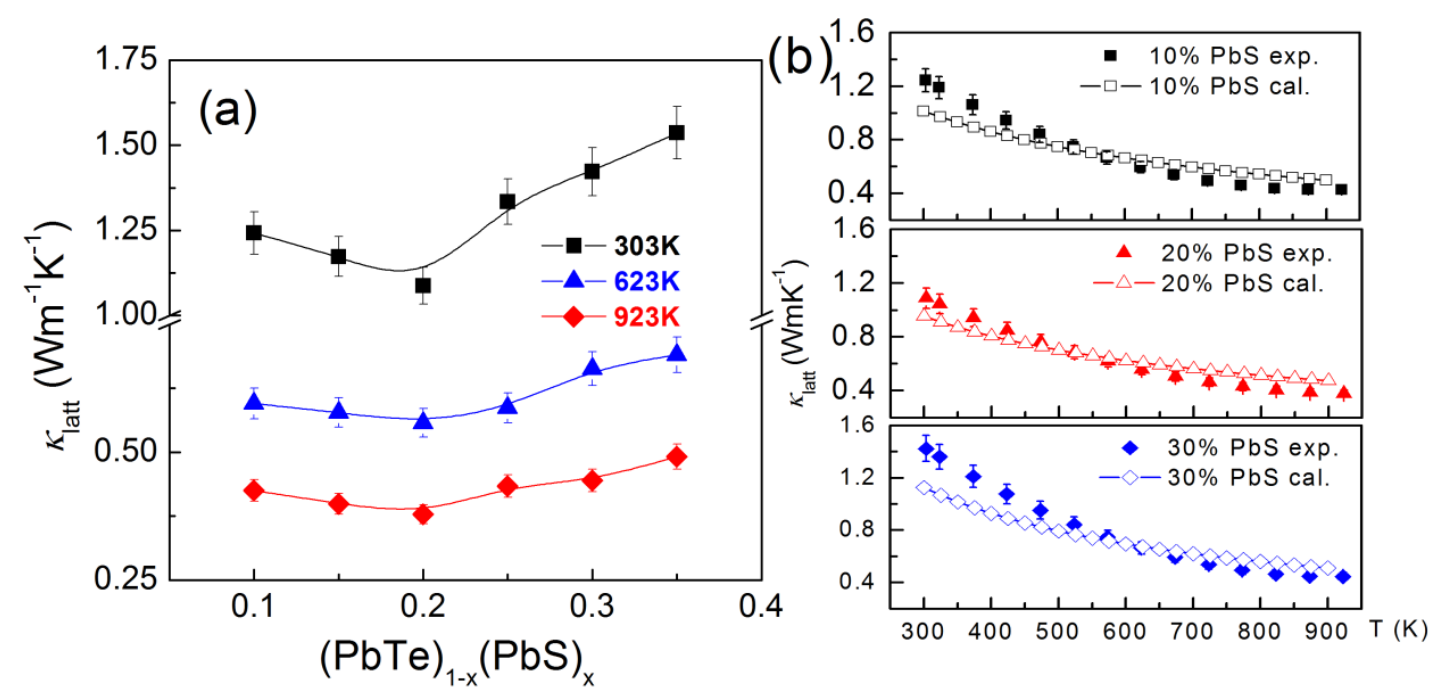

Figure 4 (a) Lattice thermal conductivities (at $303 \mathrm{~K}, 623 \mathrm{~K}$ and $923 \mathrm{~K}$ ) in $(\mathrm{PbTe})_{1-x}(\mathrm{PbS})_{x}$ samples decrease as $x$ increases from 0.1 to 0.2 , then increase as elevating $x$ value. (b) Calculated lattice thermal conductivities taking account of precipitates and alloying scattering (SI) show 
considerably match with experimental results. The data of $x=0.2$ and 0.3 was calculated based on the volume fraction of $\mathrm{PbTe}$ - and $\mathrm{PbS}$ - rich phases, with a phenomenological effective medium theory (EMT) described elsewhere ${ }^{41,42}$.

\section{Reduced thermal conductivity in SPS samples}

Girard et $a .^{21}$ have systematically studied the thermoelectric performance in Na- doped $(\mathrm{PbTe})_{1-x}(\mathrm{PbS})_{x}$ ingots, and reported very low thermal conductivity as a result of PbS alloying and Na- doping. Compared with the ingot samples (2 at $\%$ Na- doped $(\mathrm{PbTe})_{0.88}(\mathrm{PbS})_{0.12}$ was chosen due to its best thermoelectric performance as reported in ref.21), SPSed samples in this work exhibit very close electrical conductivities and Seebeck coefficients, as seen in Figure 5(a) and 5(b). Moreover, the trends of temperature dependent Hall carrier concentration $p$ and mobility $\mu$ are also consistent with those of ingots ${ }^{21}\left(\mathrm{z}\right.$ at\% Na- doped $(\mathrm{PbTe})_{0.88}(\mathrm{PbS})_{0.12}$ with $z=1.0,1.5$ and 2.0), Figure 5(c) and 5(d). The abrupt rise of $p$ and dive of $\mu$ above $650 \mathrm{~K}$ are believed to be artificial and merely the result of thermally activated intrinsic electrons from valence bands to conduction bands in both SPSed samples and ingots. Room temperature Hall coefficients are used for discussion to diminish the contribution from heavier $\Sigma$ valence bands. The room temperature solubility of Na is suggested to be $\sim 0.5 \mathrm{at} \%{ }^{39}$ or $\sim 0.4 \mathrm{at} \%{ }^{43}$ in PbTe, and $\sim 2 \mathrm{at} \%$ in $\mathrm{PbS}^{39}$; therefore, excessive Na- doping more than 0.5 at\% shall consistently lead to a hole concentration of $\sim$ $7.5 \times 10^{19} \mathrm{~cm}^{-3}\left(0.5 \% \times N_{\mathrm{A}} \rho / \mathrm{M}_{\mathrm{PbTe}}\right.$, where $N_{\mathrm{A}}$ is Avogadro's constant, $\rho$ the mass density and $\mathrm{M}_{\mathrm{PbTe}}$ the molecular mass of $\mathrm{PbTe}$ ). This value is pretty much the same as the average hole concentrations in the three $(\mathrm{PbTe})_{0.88}(\mathrm{PbS})_{0.12}-x$ at\% Na ingots, as inserted in Figure 5(c). The relatively higher hole concentrations (correspondingly lower hole mobility, as inserted in Figure 5(d)) in SPSed samples might imply the existence of extra p-type point defects, as compared with ingots. We suggest these extra p-type point defects are $\mathrm{Pb}$ vacancies, and performed Density 
Functional Theory (DFT) calculations (details in SI) to validate this assumption. Indeed, $\mathrm{Pb}$ vacancy is the defect with the lowest formation energy both in PbTe and PbS phases, which is highly possible to come from the high-energy powdering and consolidation processes. Considering the very similar microstructures between SPS samples in our work and reported ingots samples ${ }^{21}$, we believe that these extra $\mathrm{Pb}$ vacancies contribute to the consistently lowered lattice thermal conductivity in SPSed samples (Figure 5(e)), at least partly. Besides, the inevitable grain interface barriers or slight porosity coming from the powdering (hand grinding) and SPS/HP processes, shall also be responsible for the reduced lattice thermal conductivity, as widely reported in thermoelectrics, e.g., $\mathrm{PbTe}^{39,44}, \mathrm{PbSe}^{20,39,45}, \mathrm{PbS}^{2,31,46}$ and $\left(\mathrm{Zr}_{0.6} \mathrm{Hf}_{0.4}\right)_{0.9} \mathrm{Ti}_{0.1} \mathrm{NiSn}^{47}$, Figure 5(f) and Table 1. The reduced carrier mobilities seen in SPSed samples provide another circumstantial evidence for the grain interface barriers. It is just such lowered lattice thermal conductivity that results in the ZT outperformance of SPSed samples over corresponding ingots, Figure 5(h).

Table 1 Reported lattice thermal conductivity values $\kappa_{\text {latt }}$ of SPS/HP samples vs corresponding ingots. Note: only hand grindings (no ball milling) were performed before SPS/HP processes in all these cases, to exclude the grain size effect; all SPS/HP samples were highly consolidated (> 95\% at least); the unit of $\kappa_{\text {latt }}$ is in $\mathrm{Wm}^{-1} \mathrm{~K}^{-1}$.

\begin{tabular}{ccc}
\hline Compositions & $\kappa_{\text {latt }}$ ingots & $\kappa_{\text {latt }}$ SPS/HP \\
\hline PbTe & $2.2^{39}$ & $2.0^{44}$ \\
PbSe & $1.9^{39}$ & $1.6^{20,45}$ \\
$\mathrm{PbS}$ & $2.6^{2}$ & $1.5^{31,46}$ \\
$\left(\mathrm{Hf}_{0.6} \mathrm{Zr}_{0.4}\right)_{0.9} \mathrm{Ti}_{0.1} \mathrm{NiSn}$ & $3.67^{47}$ & $3.0^{47}$ \\
\hline
\end{tabular}



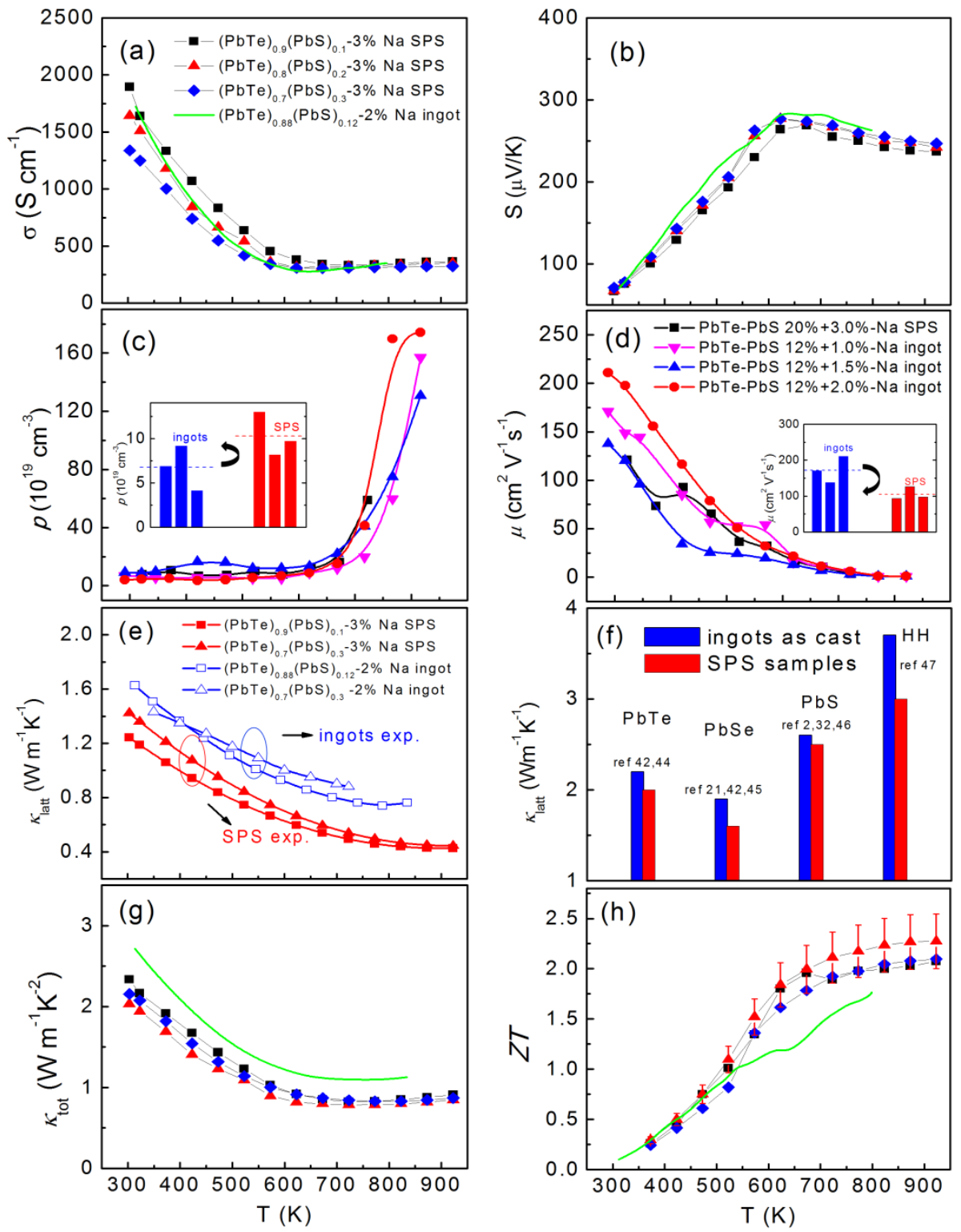

Figure 5 (a)(b) Very close electrical conductivities and Seebeck coefficients were observed in $\mathrm{PbTe}-\mathrm{PbS}$ ingots and SPSed samples. (c)(d) Average larger carrier concentration and lower mobility were found in SPSed samples, indicating a relatively higher p-type point defect level in SPSed samples. (e) Considerably lower lattice thermal conductivities were observed in SPSed samples, (f) lower lattice thermal conductivities in highly dense SPSed samples vs. ingots were also widely reported in other thermoelectric materials. (g)(h) The consistently lower lattice thermal conductivities in SPSed samples compared with ingots explain the observed superior overall ZT. For concision, error bars of ZT was implemented for the $x=20 \%$ sample.

\section{Saturation of Seebeck coefficient $S$ and electrical conductivity $\sigma$}

Obvious saturations of both Seebeck coefficients and electrical conductivities in the Na- doped SPSed $(\mathrm{PbTe})_{1-x}(\mathrm{PbS})_{x}$ samples $(x=10 \%, 20 \%$ and 30\%) started around $600 \mathrm{~K}$, as indicated by the 
arrows in Figure 6(a). Features of saturations at the similar temperature were also reported in Nadoped $(\mathrm{PbTe})_{0.88}(\mathrm{PbS})_{0.12}$ ingots by Girard et al. ${ }^{21}$, Na- doped PbTe by Aiyapetyants et al. ${ }^{48}$, Nadoped $(\mathrm{PbTe})_{1-x-y}(\mathrm{PbSe})_{x}(\mathrm{PbS})_{y}$ by Korkosz et al. ${ }^{49}$ For comparison, the K- doped $(\mathrm{PbTe})_{0.7}(\mathrm{PbS})_{0.3}$ SPSed sample ${ }^{22}$ exhibited smooth curves with elevating temperature (golden solid lines); only 3 at \% K- doped sample was included in the plots for concision. In contrast to the relatively close values of Na- and K- doped SPSed samples below $600 \mathrm{~K}$, the discrepancies of $\mathrm{S}$ and $\sigma$ at $900 \mathrm{~K}$ are as large as $25 \%$ and $50 \%$, individually. The saturations of $\mathrm{S}$ and $\sigma$ in these samples are apparently crucial to maintain the superior power factors as compared with K- doped ones, as Figure 6(b) shows. We will show later that the saturation phenomena come from the modulated hole concentration at elevated temperatures. Although the hole concentrations in different heavily doped samples vary from each other, the room temperature Seebeck coefficients are extremely close, owing to the domination of the lower heavier valence bands ( $\Sigma$-bands) in $\mathrm{PbTe}^{2,48}$, which is further verified by the calculated Pisarenko line at $303 \mathrm{~K}$ as shown in Figure 6(c). The calculations were based on the relaxation time approximated Boltzmann Transport Equation in the framework of two valence bands ${ }^{9,50}$, i.e., upper lighter valence bands ( $L$-bands) and $\Sigma$-bands.

For heavily doped p-type PbTe, the saturation of $\mathrm{S}$ and $\sigma$ was previously ascribed by Airapetyants $^{48}$ to the thermally excited electrons from valence to conduction bands, i.e., bipolar effect, probably due to the coincidence of their onset temperatures. Nevertheless, this explanation was challenged by follow-up experimental values ${ }^{9,51}$ and calculations ${ }^{9,52}$ (including our own calculations). The concentration of thermally activated electrons at $600 \mathrm{~K}$ is calculated to be only around $\sim 10^{16} \mathrm{~cm}^{-3}$, Figure 6(d), which is obviously insignificant compared with the majority carrier (hole) concentration $\sim 10^{19} \mathrm{~cm}^{-3}$; these values are found to be consistent with calculations 
reported elsewhere ${ }^{53}$. Korkosz et al. ${ }^{49}$ recently suggested that the saturation of $\mathrm{S}$ and $\sigma$ could be explained by the topological electrons, the notion of which was proposed previously to explain the observed negative low-field Hall coefficients $\mathrm{R}_{\mathrm{H}}$ in Na- doped PbTe-PbS samples at $\sim 600 \mathrm{~K}^{53}$. When the initially separated $L$-pockets and $\Sigma$-pockets singly connect with a continuous Fermi surface, a negative curvature would form at the "neck" of connection. The charge carriers at that "neck" region behave like electrons in response to a potential or a temperature gradient, and contribute negatively to the Seebeck coefficient opposite to the majority charge carriers (holes). However, this explanation might not suit our case well, since both literatures ${ }^{21}$ and our own Hall measurements of similar compositions show positive Hall coefficients as high as $823 \mathrm{~K}$, in contrast to the reported negative $\mathrm{R}_{\mathrm{H}}$ at $\sim 600 \mathrm{~K}$. It was also argued ${ }^{49}$ that sulfur helps for the formation of the topological connection of $L$ and $\Sigma$ bands and is thus responsible for the onset temperature of saturation; the reason was that sulfur $p$-orbitals could lower the $L$ bands thus narrowed the energy gap between $L$ and $\Sigma$ bands, resulting in the contribution of topological electrons to the overall electrical transport at a lower temperature. In our understandings, since PbS has a wider band gap and larger energy offset between $L-\Sigma$ band edges compared with $\mathrm{PbTe}^{4}$, the alloying of $\mathrm{PbS}$ with PbTe would also lower the energy levels of $\Sigma$ bands; meanwhile, observations ${ }^{53}$ didn’t show a stronger sign of electron-like behavior in $12 \%$ sulfur sample than in the $8 \%$ sulfur one. What is more, the topological electrons result from the "touch" of the Fermi surface of $L$ and $\Sigma$ bands, thus are related only with material's intrinsic band structures; however, difficulties were faced when one tried to explain the disparate electrical behaviors in Na- (obvious saturations) and $\mathrm{K}$ - heavily doped (no saturations were observed) $(\mathrm{PbTe})_{0.7}(\mathrm{PbS})_{0.3}{ }^{22}$, and the distinguishing trends of $\mathrm{S}$ and $\sigma$ in Na-doped $(\mathrm{PbTe})_{1-x-y}(\mathrm{PbSe})_{x}(\mathrm{PbS})_{y}$ during heating and cooling cycles ${ }^{49}$. 
It is interesting to notice that most (if not all) of the saturation phenomena took place in Nadoped samples, such as Na- doped $(\mathrm{PbTe})_{0.88}(\mathrm{PbS})_{0.12}$ by Girard et al. ${ }^{21}$, Na-doped $(\mathrm{PbTe})_{1-\mathrm{x}-\mathrm{y}}$ $(\mathrm{PbSe})_{\mathrm{x}}(\mathrm{PbS})_{\mathrm{y}}$ by Korkosz et al. ${ }^{49}$ and Na-doped $\mathrm{Pb}_{0.96} \mathrm{Sr}_{0.04} \mathrm{Te}$ by Biswas et al. ${ }^{14}$ Especially, comparing $\mathrm{K}$ - doped $(\mathrm{PbTe})_{0.7}(\mathrm{PbS})_{0.3}$ samples $^{22}$ with Na- doped ones in this work (note that their synthesis processes are exactly the same, and that the hole concentrations at room temperatures are close too), Figure 5(a) and 5(b), it is natural to argue that sodium is one of the key factors for the observed saturation features. Another common place of the Na- doped samples which exhibited such saturation features is the existence of vast grain/phase boundaries or dislocations. It was previously evidenced that excessive Na beyond the solubility limit tends to segregate at these grain/phase boundaries by Biswas et al. ${ }^{14}$ and $\mathrm{He}$ et $a l .^{26}$, and that they may liquefy at temperatures above $633 \mathrm{~K}$ by Yamini et al. ${ }^{54}$ Herein, we suggest that the saturation of $\mathrm{S}$ and $\sigma$ results from the diffusion and re-dissolution of $\mathrm{Na}$, which was confined to grain/phase boundaries at low temperature, back into the matrix grains at elevated temperature. Na's re-dissolution as p-type doping would increase the matrix's hole concentration, leading to a decreased Seebeck coefficient and increased electrical conductivity. We conducted electrical transport calculations from solving the Boltzmann Transport Equation accounting for the contribution from three related bands $^{9,50}$ ( $L+\Sigma$ valence bands along with $C$ conduction band) to validate our suggestion, details can be found in SI. As seen in Figure 6(e) and 6(f), electrical conductivity $\sigma$ and Seebeck coefficient S vary systematically with the elevating hole concentration, from $0.8 \times 10^{20}$ to $1.6 \times 10^{20}$ $\mathrm{cm}^{-3} \cdot 0.8 \times 10^{20} \mathrm{~cm}^{-3}$ represents the measured room temperature Hall coefficient, while $1.6 \times 10^{20}$ $\mathrm{cm}^{-3}$ corresponds to the high temperature solution limit of $\mathrm{Na}$ in PbTe system ${ }^{43}$. The onset temperature of Na's diffusion at $600 \mathrm{~K}$ probably imply a potential barrier $\sim 0.052 \mathrm{eV}$. For a better 
illustration, we put the calculated curves of increasing hole concentration together with those calculated with a constant hole concentration $\left(0.8 \times 10^{20} \mathrm{~cm}^{-3}\right)$, and compare them with experimental data of 3 at\% Na- doped $(\mathrm{PbTe})_{1-x}(\mathrm{PbS})_{x}(x=10 \%, 20 \%$ and 30\%), Figure 6(g) and 6(h). If the carrier concentration is kept constant, $\mathrm{S}$ tends to increase smoothly to the peak around $750 \mathrm{~K}$, whereafter it starts to dive due to the bipolar effect. The discrepancy between the calculation with fixed hole concentration and experimental results again proves the deficiency of Airapetyants's suggestion. In contrast, calculations with an increasing hole concentration match the experiments pretty well. The modulation of hole concentration due to Na's diffusion at elevated temperature (over $\sim 600 \mathrm{~K}$ ) is beneficial to the overall thermoelectric performance; this can be understood in the viewpoint of tuning the actual carrier concentration closer to the temperature dependent optimized concentration as argued by Pei et al. ${ }^{55}$, as Figure S2 shows. At last, it is also understandable that no obvious saturations were found in $\mathrm{K}$ - heavily doped $(\mathrm{PbTe})_{0.7}(\mathrm{PbS})_{0.3}$, since the radius of $\mathrm{K}^{+}(\sim 0.133 \mathrm{~nm})$ is much larger than that of $\mathrm{Na}^{+}(\sim 0.095 \mathrm{~nm})$ and it is hard for $\mathrm{K}^{+}$to diffuse into matrix as $p$-type dopant at elevated temperatures.

We cannot exclude the possibility that topological electrons and bipolar effect might also contribute to the observed saturation of S and $\sigma$ at high temperatures, but they don't necessarily play such a dominant role as suggested. Overall, the underlying reason is complicated and could be the combination of elevated solubility of $\mathrm{Na}$ (dominating), topological electrons $\mathrm{s}^{49,53}$ and intrinsic electrons ${ }^{48}$; the latter two factors might only function at even higher temperatures. 

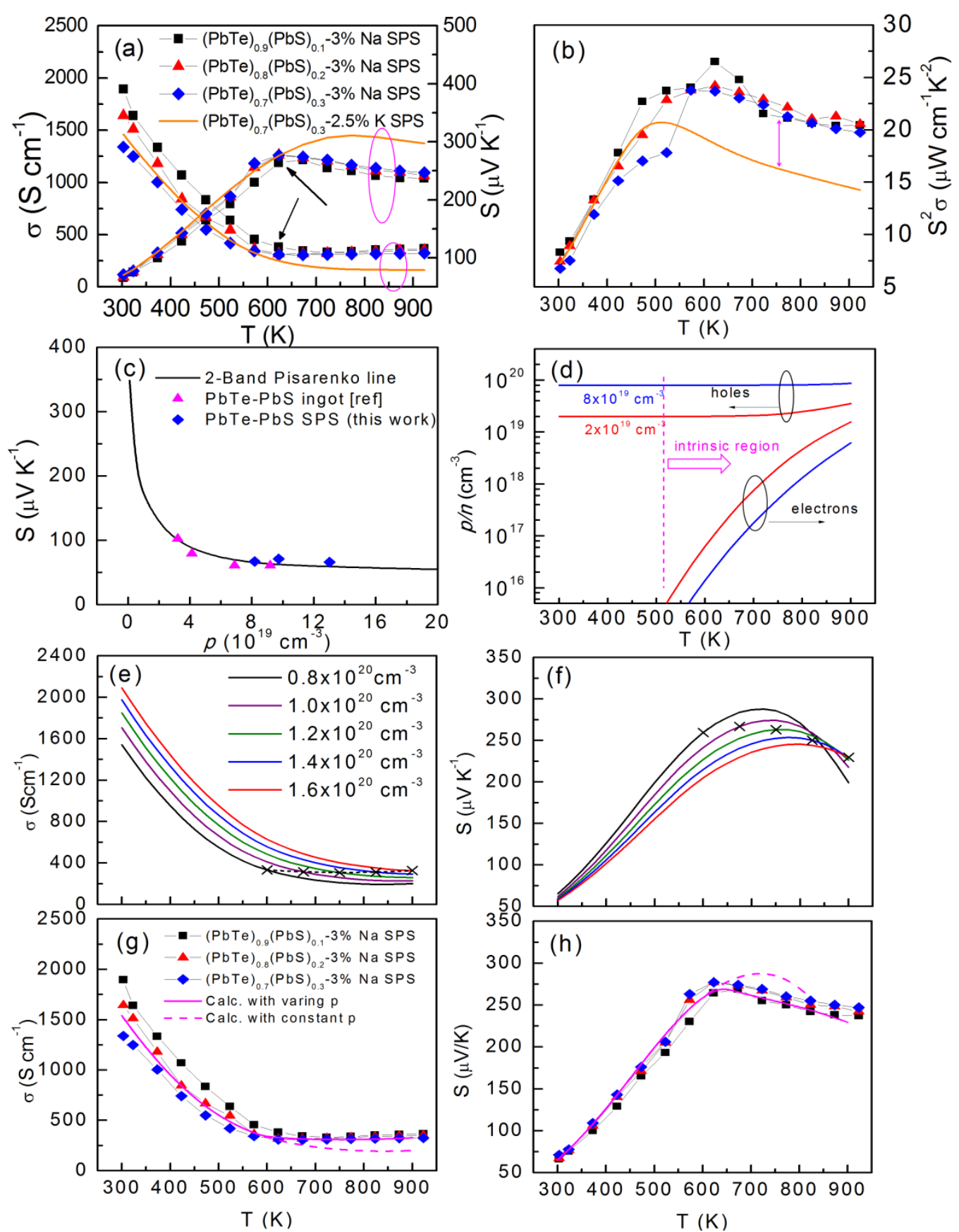

Figure 6 (a)(b) Obvious saturation occurs in both electrical conductivity and Seebeck coefficient in Na- doped PbTe-PbS composites over $\sim 600 \mathrm{~K}$, in contrast to K- doped ones. (c) The similarity of low temperature Seebeck values between Na- and K- doped SPSed samples can be explained as the contribution from the lower heavy valence bands, as the calculated Pisarenko line (303K) shows. (d) The saturation at $\sim 600 \mathrm{~K}$ cannot be well explained by the thermally activated intrinsic electrons. (e)(f) A systematical calculation of electrical conductivity and Seebeck coefficient as hole concentration increases from $0.8 \times 10^{20}$ to $1.6 \times 10^{20} \mathrm{~cm}^{-3}$. (g)(h) Calculation with constant hole concentration and intrinsic electrons cannot explain the experiments, while that of increasing hole concentration can well fit measured data. 


\section{Conclusions}

PbTe-PbS pseudo-binary owns a complex microstructure due to the existence of immiscible gap between PbTe and PbS phases, thus exhibiting very low thermal conductivity as compared with its end members PbTe and PbS. We have synthesized and systematically studies 3 at\% Nadoped $(\mathrm{PbTe})_{1-x}(\mathrm{PbS})_{x}$ SPSed samples with varying PbS phase fractions $x(x=10 \%, 15 \%, 20 \%$, 25\%, 30\% and 35\%). An outstanding figure of merit $Z T \sim 2.3$ was obtained at $923 \mathrm{~K}$ when $\mathrm{PbS}$ phase fraction is at $20 \%$, corresponding to the lowest lattice thermal conductivity. The relation between microstructure and lattice thermal conductivity was then discussed based on TEM observations and theoretical calculations. Besides, we ascribed the consistently lower thermal conductivities in SPSed samples than those in ingots to the powdering and consolidation processes. The hole concentration modulation as a result of excessive Na diffusion and re-dissolution into the matrix grains was suggested to be responsible for the observed saturations of electrical properties at $\sim 600 \mathrm{~K}$, which phenomena favorably result in the superior power factors in Na- doped samples than in K- doped ones.

\section{Sample synthesis and characterizations}

Sample synthesis: Ingots $(\sim 20 \mathrm{~g})$ with nominal compositions of $(\mathrm{PbTe})_{1-x}(\mathrm{PbS})_{x}-3$ at $\% \mathrm{Na}(x=$ $10 \%, 15 \%, 20 \%, 25 \%, 30 \%$ and $35 \%$ ) were synthesized by mixing appreciated ratios of high purity raw materials of $\mathrm{Pb}, \mathrm{Te}, \mathrm{S}$ and $\mathrm{Na}$ in carbon-coated quartz tubes in an $\mathrm{N}_{2}$-filled glove box. The raw materials used are as follows: $\mathrm{Pb}$ wire (99.99\%, American Elements, US), Te shot (99.999\%, 5N Plus, Canada), S shot or chunk (99.999\%, 5N Plus, Canada) and Na chunk (99.999\%, Sigma-Aldrich, US). The quartz tubes were then evacuated to a pressure of $\sim 10^{-4}$ torr, 
flame-sealed, melted at 1,323 K over 10h, and quenched to room temperature (297 K). The obtained ingots were crushed into powders and then densified by spark plasma sintering (SPS) method (SPS-211Lx, Dr. Sinter), highly dense samples can achieve $>97 \%$ of theoretical density. To prepare for SPS processing, the melt grown ingots were first hand ground into powders $(<5$ $\mathrm{mm}^{3}$ ) with mortar and pestle and then further ground by a mechanical mortar and pestle to reduce the grains to less than $53 \mu^{3}$. These powders were then densified at $823 \mathrm{~K}$ for $10 \mathrm{~min}$ in a $20 \mathrm{~mm}$ diameter graphite die under an axial compressive stress of $40 \mathrm{MPa}$. Highly dense disk-shaped pellets with dimensions of $\varnothing 20 \mathrm{~mm} \times 9 \mathrm{~mm}$ were thus obtained. The manipulations and preparation steps for powders grinding were carried out in a glove box of purified $\mathrm{N}_{2}$-atmosphere.

Electrical properties: The obtained pellets were cut into bars with dimensions of $18 \mathrm{~mm} \times 3$ $\mathrm{mm} \times 3 \mathrm{~mm}$ that and used for simultaneous Seebeck coefficient and electrical conductivity measurements with an Ulvac Riko ZEM-3 instrument under a He-atmosphere from room temperature to $923 \mathrm{~K}$. The samples were coated with a thin layer $(0.1-0.2 \mathrm{~mm})$ of boron nitride (BN) to protect instruments from contamination. Heating and cooling cycles gave reproducible electrical properties thus verified these samples were thermally stable. The uncertainty of the Seebeck coefficient and electrical conductivity measurements is $\sim 5 \%$. The Hall coefficients were measured using the Van der Pauw technique under a reversible magnetic field of 0.52 T (8340DC, Toyo, Japan), and the uncertainty is estimated to be within $5 \%$.

Thermal conductivity: High density SPS processed pellets were cut and polished into coins of $\varnothing \sim 8 \mathrm{~mm}$ and thickness $\sim 1-2 \mathrm{~mm}$ for thermal diffusivity measurements. The samples were coated with a thin layer of graphite to minimize errors from the emissivity of the materials. The thermal conductivity was calculated from $\kappa=\mathrm{D} \cdot \mathrm{C}_{p} \cdot \rho$, where the thermal diffusivity coefficient (D) was 
measured using the laser flash diffusivity method in a Netzsch LFA457, the specific heat capacity $\left(C_{p}\right)$ was indirectly derived using a representative sample (Pyroceram 9606) in the temperature range 300-923K, and the density $(\rho)$ was determined using the dimensions and mass of the sample, and reconfirmed using a gas pycnometer (Micromeritics AccuPyc1340) measurement. The thermal diffusivity data were analyzed using a Cowan model with pulse correction; heating and cooling cycles gave reproducible values for every individual sample. The uncertainty of the thermal conductivity is estimated to be within $5 \%$, considering the uncertainties for $\mathrm{D}, \mathrm{C}_{p}$ and $\rho$. The combined uncertainty for all measurements involved in the calculation of ZT is about $12 \%$.

Electron Microscopy: S/TEM investigations were carried out in an aberration corrected JEOL ARM200 microscope operated at $200 \mathrm{kV}$. The thin TEM specimens were prepared by conventional standard methods. The procedures include cutting, grinding, dimpling, polishing and Ar-ion milling with a liquid nitrogen cooling stage.

\section{Acknowledgements}

This contribution was supported by the startup of South University of Science and Technology of China, and partly supported by Science, Technology and Innovation Commission of Shenzhen Municipality (Grant No. JCYJ20140612140151884), the “zhuoyue program” from Beihang University (LDZ), the NSFC under grant No. 51202008 and Postdoctoral Science Foundation of China (2013M540037) (Y.L.P). D. Wu would like to thank Haijun Wu and Fengshan Zheng for their thoughtful discussions on this manuscript. The Work at BNL was supported by the U.S.

Department of Energy, Office of Basic Energy Science, Material Science and Engineering

Division, under Contract No. DE-AC02-98CH10886. 


\section{Reference}

(1) Rowe, D. M. CRC handbook of thermoelectrics; CRC press, 1995.

(2) Ravich, Y. I. Semiconducting Lead Chalcogenides (Plenum). 1970.

(3) Pei, Y.; Wang, H.; Snyder, G. J. Advanced materials 2012, 24, 6125.

(4) Gibbs, Z. M.; Kim, H.; Wang, H.; White, R. L.; Drymiotis, F.; Kaviany, M.; Jeffrey Snyder, G. Applied Physics Letters 2013, 103, 262109.

(5) Božin, E. S.; Malliakas, C. D.; Souvatzis, P.; Proffen, T.; Spaldin, N. A.; Kanatzidis, M. G.; Billinge, S. J. Science 2010, 330, 1660.

(6) Delaire, O.; Ma, J.; Marty, K.; May, A. F.; McGuire, M. A.; Du, M. H.; Singh, D. J.; Podlesnyak, A.; Ehlers, G.; Lumsden, M. D.; Sales, B. C. Nat. Mater. 2011, 10, 614.

(7) Heremans, J. P.; Jovovic, V.; Toberer, E. S.; Saramat, A.; Kurosaki, K.; Charoenphakdee, A.; Yamanaka, S.; Snyder, G. J. Science 2008, 321, 554.

(8) Heremans, J. P.; Wiendlocha, B.; Chamoire, A. M. Energy \& Environmental Science 2012, 5, 5510.

(9) Pei, Y.; Shi, X.; LaLonde, A.; Wang, H.; Chen, L.; Snyder, G. J. Nature 2011, 473, 66.

(10) Zhao, L. D.; Wu, H. J.; Hao, S. Q.; Wu, C. I.; Zhou, X. Y.; Biswas, K.; He, J. Q.; Hogan, T. P.; Uher, C.; Wolverton, C.; Dravid, V. P.; Kanatzidis, M. G. Energy \& Environmental Science 2013, 6, 3346.

(11) Wang, H.; Wang, J.; Cao, X.; Snyder, G. J. Journal of Materials Chemistry A 2014, 2, 3169.

(12) Pei, Y.; Wang, H.; Gibbs, Z. M.; LaLonde, A. D.; Snyder, G. J. NPG Asia Materials 2012, 4, e28.

(13) He, J.; Kanatzidis, M. G.; Dravid, V. P. Materials Today 2013, 16, 166.

(14) Biswas, K.; He, J.; Blum, I. D.; Wu, C. I.; Hogan, T. P.; Seidman, D. N.; Dravid, V. P.; Kanatzidis, M. G. Nature 2012, 489, 414.

(15) Pei, Y.; Lensch-Falk, J.; Toberer, E. S.; Medlin, D. L.; Snyder, G. J. Advanced Functional Materials 2011, 21, 241.

(16) Martin, J.; Nolas, G.; Zhang, W.; Chen, L. Applied physics letters 2007, 90, 222112.

(17) Wu, H.; Carrete, J.; Zhang, Z.; Qu, Y.; Shen, X.; Wang, Z.; Zhao, L.-D.; He, J. NPG Asia Materials 2014, 6, e108.

(18) Kuo, C.-H.; Chien, H.-S.; Hwang, C.-S.; Chou, Y.-W.; Jeng, M.-S.; Yoshimura, M. Materials transactions 2011, 52, 795.

(19) Wang, J. L.; Wang, H.; Snyder, G. J.; Zhang, X.; Ni, Z. H.; Chen, Y. F. Journal of Physics D: Applied Physics 2013, 46, 405301.

(20) Zhang, Q.; Cao, F.; Liu, W.; Lukas, K.; Yu, B.; Chen, S.; Opeil, C.; Broido, D.; Chen, G.; Ren, Z. Journal of the American Chemical Society 2012, 134, 10031.

(21) Girard, S. N.; He, J.; Zhou, X.; Shoemaker, D.; Jaworski, C. M.; Uher, C.; Dravid, V. P.; Heremans, J. P.; Kanatzidis, M. G. Journal of the American Chemical Society 2011, 133, 16588.

(22) Wu, H. J.; Zhao, L. D.; Zheng, F. S.; Wu, D.; Pei, Y. L.; Tong, X.; Kanatzidis, M. G.; He, J. Q. Nature communications 2014, 5, 4515.

(23) Androulakis, J.; Todorov, I.; He, J.; Chung, D. Y.; Dravid, V.; Kanatzidis, M. Journal of the American Chemical Society 2011, 133, 10920.

(24) Leute, V.; Volkmer, N. Zeitschrift für Physikalische Chemie 1985, 144, 145.

(25) Girard, S. N.; He, J.; Li, C.; Moses, S.; Wang, G.; Uher, C.; Dravid, V. P.; Kanatzidis, M. G. Nano letters 2010, 10, 2825 .

(26) He, J.; Blum, I. D.; Wang, H. Q.; Girard, S. N.; Doak, J.; Zhao, L. D.; Zheng, J. C.; Casillas, G.; 
Wolverton, C.; Jose-Yacaman, M.; Seidman, D. N.; Kanatzidis, M. G.; Dravid, V. P. Nano letters 2012, 12, 5979.

(27) Girard, S. N.; Schmidt-Rohr, K.; Chasapis, T. C.; Hatzikraniotis, E.; Njegic, B.; Levin, E. M.; Rawal, A.; Paraskevopoulos, K. M.; Kanatzidis, M. G. Advanced Functional Materials 2013, 23, 747.

(28) He, J.; Girard, S. N.; Kanatzidis, M. G.; Dravid, V. P. Advanced Functional Materials 2010, 20, 764.

(29) Wang, H.; Bahk, J. H.; Kang, C.; Hwang, J.; Kim, K.; Kim, J.; Burke, P.; Bowers, J. E.; Gossard, A. C.; Shakouri, A.; Kim, W. Proceedings of the National Academy of Sciences of the United States of America 2014, 111, 10949.

(30) Johnsen, S.; He, J.; Androulakis, J.; Dravid, V. P.; Todorov, I.; Chung, D. Y.; Kanatzidis, M. G. Journal of the American Chemical Society 2011, 133, 3460.

(31) Wang, H.; Schechtel, E.; Pei, Y.; Snyder, G. J. Advanced Energy Materials 2013, 3, 488.

(32) Rowe, D. M.; Bhandari, C. Modern thermoelectrics; Prentice Hall, 1983.

(33) Wu, D.; Pei, Y.; Wang, Z.; Wu, H.; Huang, L.; Zhao, L.-D.; He, J. Advanced Functional Materials 2014, 24, 7763.

(34) Koh, Y. K.; Vineis, C.; Calawa, S.; Walsh, M.; Cahill, D. G. Applied Physics Letters 2009, 94, 153101.

(35) Hsu, K. F.; Loo, S.; Guo, F.; Chen, W.; Dyck, J. S.; Uher, C.; Hogan, T.; Polychroniadis, E. K.; Kanatzidis, M. G. Science 2004, 303, 818.

(36) Zhao, L.-D.; Lo, S.-H.; Zhang, Y.; Sun, H.; Tan, G.; Uher, C.; Wolverton, C.; Dravid, V. P.; Kanatzidis, M. G. Nature 2014, 508, 373.

(37) Doak, J. W.; Wolverton, C. Physical Review B 2012, 86, 144202.

(38) He, J.; Androulakis, J.; Kanatzidis, M. G.; Dravid, V. P. Nano letters 2012, 12, 343.

(39) He, J.; Zhao, L. D.; Zheng, J. C.; Doak, J. W.; Wu, H.; Wang, H. Q.; Lee, Y.; Wolverton, C.; Kanatzidis, M. G.; Dravid, V. P. Journal of the American Chemical Society 2013, 135, 4624.

(40) Medlin, D. L.; Snyder, G. J. Current Opinion in Colloid \& Interface Science 2009, 14, 226.

(41) Minnich, A.; Chen, G. Applied Physics Letters 2007, 91, 073105.

(42) Wu, D.; Petersen, A. S.; Poon, S. J. AIP Advances 2013, 3, 082116.

(43) Yamini, S. A.; Ikeda, T.; Lalonde, A.; Pei, Y.; Dou, S. X.; Snyder, G. J. Journal of Materials Chemistry A 2013, 1, 8725.

(44) LaLonde, A. D.; Pei, Y.; Snyder, G. J. Energy \& Environmental Science 2011, 4, 2090.

(45) Wang, H.; Pei, Y.; LaLonde, A. D.; Snyder, G. J. Proceedings of the National Academy of Sciences 2012, 109, 9705.

(46) Zhao, L. D.; Lo, S. H.; He, J.; Li, H.; Biswas, K.; Androulakis, J.; Wu, C. I.; Hogan, T. P.; Chung, D. Y.; Dravid, V. P.; Kanatzidis, M. G. Journal of the American Chemical Society 2011, 133, 20476.

(47) Kurosaki, K.; Maekawa, T.; Muta, H.; Yamanaka, S. Journal of Alloys and Compounds 2005, $397,296$.

(48) Airapetyants, S.; Vinogradova, M.; Dubrovskaya, I.; Kolomoets, N.; Rudnik, I. SOVIET PHYS SOLID STATE 1966, 8, 1069.

(49) Korkosz, R. J.; Chasapis, T. C.; Lo, S. H.; Doak, J. W.; Kim, Y. J.; Wu, C. I.; Hatzikraniotis, E.; Hogan, T. P.; Seidman, D. N.; Wolverton, C.; Dravid, V. P.; Kanatzidis, M. G. Journal of the American Chemical Society 2014, 136, 3225.

(50) Wu, D.; Zhao, L. D.; Hao, S.; Jiang, Q.; Zheng, F.; Doak, J. W.; Wu, H.; Chi, H.; Gelbstein, Y.; Uher, C.; Wolverton, C.; Kanatzidis, M.; He, J. Journal of the American Chemical Society 2014, 136, 
11412.

(51) Pei, Y.; LaLonde, A.; Iwanaga, S.; Snyder, G. J. Energy \& Environmental Science 2011, 4, 2085.

(52) Pei, Y.; Wang, H.; Snyder, G. J. Advanced materials 2012, 24, 6125.

(53) Jaworski, C. M.; Nielsen, M. D.; Wang, H.; Girard, S. N.; Cai, W.; Porter, W. D.; Kanatzidis, M. G.; Heremans, J. P. Physical Review B 2013, 87.

(54) Yamini, S. A.; Ikeda, T.; Lalonde, A.; Pei, Y.; Dou, S. X.; Snyder, G. J. Journal of Materials Chemistry A 2013, 1, 8725.

(55) Pei, Y.; LaLonde, A. D.; Heinz, N. A.; Shi, X.; Iwanaga, S.; Wang, H.; Chen, L.; Snyder, G. J. Advanced materials 2011, 23, 5674. 